\title{
EMPTY PENTAGONS IN POINT SETS WITH COLLINEARITIES
}

\author{
JÁNOS BARÁT, VIDA DUJMOVIĆ, GWENAËL JORET, MICHAEL S. PAYNE, \\ LUDMILA SCHARF, DARIA SCHYMURA, PAVEL VALTR, AND DAVID R. WOOD
}

\begin{abstract}
An empty pentagon in a point set $P$ in the plane is a set of five points in $P$ in strictly convex position with no other point of $P$ in their convex hull. We prove that every finite set of at least $328 \ell^{2}$ points in the plane contains an empty pentagon or $\ell$ collinear points. This is optimal up to a constant factor since the $(\ell-1) \times(\ell-1)$ grid contains no empty pentagon and no $\ell$ collinear points. The previous best known bound was doubly exponential.
\end{abstract}

\section{INTRODUCTION}

The Erdős-Szekeres Theorem [5], a classical result in discrete geometry, states that for every integer $k$ there is a minimum integer $\mathrm{ES}(k)$ such that every set of at least $\mathrm{ES}(k)$ points in general position in the plane contains $k$ points in convex position. Erdős [4] asked whether a similar result held for empty $k$-gons ( $k$ points in convex position with no other points inside their convex hull). Horton [9] answered this question in the negative by showing that there are arbitrarily large point sets in general position that contain no empty heptagon. On the other hand, Harborth [8] showed that every set of at least 10 points in general position contains an empty pentagon. More recently, Nicolás [11] and Gerken [7] independently settled the question for $k=6$ by showing that sufficiently large point sets in general position always contain empty hexagons; see also [10, 13].

These questions are not interesting if the general position condition is abandoned completely, since a collinear point set contains no three points in convex position.

Date: October 30, 2018.

2000 Mathematics Subject Classification. 52C10 Erdős problems and related topics of discrete geometry.

This research was supported by the DAAD and the Go8 within the Australia-Germany Joint Research Co-operation Scheme 2011/12 as part of the project Problems in geometric graph theory (Kennz. 50753217).

János Barát is supported by the Hungarian National Science Foundation (OTKA) Grant K 76099, and Australian Research Council (ARC) grant DP120100197. Vida Dujmović is supported by the Natural Sciences and Engineering Research Council (NSERC) of Canada, and by an Endeavour Fellowship from the Australian Government. Gwenaël Joret is a Postdoctoral Researcher of the Fonds National de la Recherche Scientifique (F.R.S.-FNRS), and is also supported by an Endeavour Fellowship. Michael Payne is supported by an Australian Postgraduate Award from the Australian Government. Ludmila Scharf is supported by the German Research Foundation (DFG) Grant AL 253/7-1. Daria Schymura was supported by the DFG within the Priority Programme 1307 Algorithm Engineering. Pavel Valtr is supported by the Ministry of Education of the Czech Republic under project CE-ITI (GAČR P2020/12/G061). David Wood is supported by a QEII Research Fellowship from the ARC. 
However, considering point sets with a bounded number of collinear points does lead to interesting generalisations of these problems. First some definitions are needed. A point set $X$ in the plane is in weakly convex position if every point in $X$ lies on the boundary of $\operatorname{conv}(X)$, the convex hull of $X$. A point $x \in X$ is a corner of $X$ if $\operatorname{conv}(X \backslash\{x\}) \neq \operatorname{conv}(X)$. The set $X$ is in strictly convex position if every point in $X$ is a corner of $X$. A weakly (respectively strictly) convex $k$-gon is a set of $k$ points in weakly (respectively strictly) convex position. It is well known that the Erdős-Szekeres theorem generalises for point sets with bounded collinearities; see [1] for proofs. One generalisation states that every set of at least $\operatorname{ES}(k)$ points contains a weakly convex $k$-gon. For strictly convex position, the generalisation states that for all integers $k$ and $\ell$ there exists a minimum integer $\operatorname{ES}(k, \ell)$ such that every set of at least $\operatorname{ES}(k, \ell)$ points in the plane contains $\ell$ collinear points or a strictly convex $k$-gon.

This paper addresses the case of empty pentagons in point sets with collinearities. A subset $X$ of a point set $P$ is an empty $k$-gon if $X$ is a strictly convex $k$-gon and $P \cap \operatorname{conv}(X)=X$. Abel et al. [1] showed that every finite set of at least $\operatorname{ES}\left(\frac{(2 \ell-1)^{\ell}-1}{2 \ell-2}\right)$ points in the plane contains an empty pentagon or $\ell$ collinear points. The function $\operatorname{ES}(k)$ is known to grow exponentially [5, 6], so this bound is doubly exponential in $\ell$. See [3, 12] for more on point sets with no empty pentagon. In the present paper the following theorem is proved without applying the Erdős-Szekeres Theorem.

Theorem 1. Let $P$ be a finite set of points in the plane. If $P$ contains at least $328 \ell^{2}$ points, then $P$ contains an empty pentagon or $\ell$ collinear points.

This quadratic bound is optimal up to a constant factor since the $(\ell-1) \times(\ell-1)$ square grid has $(\ell-1)^{2}$ points and contains neither an empty pentagon nor $\ell$ collinear points.

Concerning the general question of the existence of empty $k$-gons in point sets with collinearities, Horton's negative result for empty heptagons also applies in this setting. However, it is not clear how to adapt the proofs of Nicolás and Gerken to deal with collinearities, and the case $k=6$ remains open.

The point set $P$ will be assumed to be finite throughout this paper, and indeed Theorem 1 does not hold for infinite sets. A countably infinite point set in general position with no empty pentagons can be constructed recursively from any finite set in general position by repeatedly placing points inside every empty pentagon, avoiding collinearities. On the other hand, Theorem 1 easily generalises to locally finite point sets, point sets which contain only finitely many points in any bounded region. The result of Abel et al. [1] already implies that an infinite locally finite set with no empty pentagon contains $\ell$ collinear points for every positive integer $\ell$.

The remainder of this section introduces terminology that is used throughout the paper. The convex layers $L_{1}, \ldots, L_{r}$ of $P$ are defined recursively as follows: $L_{i}$ is the subset of $P$ lying in the boundary of the convex hull of $P \backslash \bigcup_{j=1}^{i-1} L_{j}$, and $L_{r}$ is the innermost layer, so $P=\bigcup_{i=1}^{r} L_{i}$ and $L_{i} \neq \emptyset$ for $i=1, \ldots, r$. Note that each layer is in weakly convex position. 

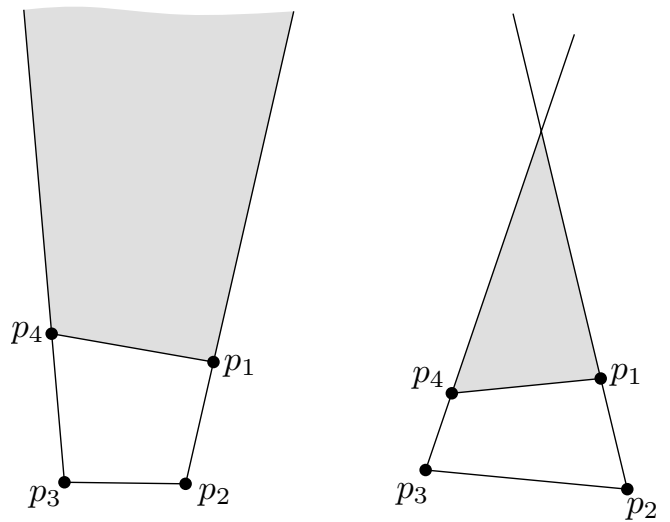

Figure 1. The shaded regions represent the 4 -sector $S\left(p_{1}, p_{2}, p_{3}, p_{4}\right)$, which may be bounded or unbounded.

Points of $P$ will also be referred to as vertices and line segments connecting two points of $P$ as edges. The edges of a layer are the edges between consecutive points in the boundary of the convex hull of that layer. Edges of layers will always be specified in clockwise order. A single letter such as $e$ is often used to denote an edge. For an edge $e$, let $l(e)$ denote the line containing $e$. Some edges will be used to determine half-planes. The open half-planes determined by $l(e)$ will be denoted $e^{+}$and $e^{-}$, where the + and - sides will be determined later. Similarly, the closed half-planes determined by $l(e)$ will be denoted $e^{\oplus}$ and $e^{\ominus}$.

Gerken [7] introduced the notion of $k$-sectors. If $p_{1} p_{2} p_{3} p_{4}$ is a strictly convex quadrilateral (that is, a strictly convex 4 -gon), then the 4 -sector $S\left(p_{1}, p_{2}, p_{3}, p_{4}\right)$ is the set of all points $q$ such that $q p_{1} p_{2} p_{3} p_{4}$ is a strictly convex pentagon. Note that the order of the arguments is significant. $S\left(p_{1}, p_{2}, p_{3}, p_{4}\right)$ is the intersection of three open half-planes, and may be bounded or unbounded, as shown in Figure 1 . The closure of a 4 -sector will be denoted by square brackets, $S\left[p_{1}, p_{2}, p_{3}, p_{4}\right]$. If $P$ contains no empty pentagon and $p_{1} p_{2} p_{3} p_{4}$ is an empty quadrilateral in $P$, then $P \cap S\left(p_{1}, p_{2}, p_{3}, p_{4}\right)=\emptyset$. Otherwise, since $P$ is finite, there exists a point $x \in P \cap S\left(p_{1}, p_{2}, p_{3}, p_{4}\right)$ closest to the line $l\left(p_{1} p_{4}\right)$, and $x p_{1} p_{2} p_{3} p_{4}$ is an empty pentagon.

\section{LARGE SUBSETS IN WEAKLY CONVEX POSITION}

The first major step in proving Theorem 1 is to establish the following theorem concerning point sets with large subsets in weakly convex position.

Theorem 2. If a point set $P$ contains $8 \ell$ points in weakly convex position, then $P$ contains an empty pentagon or $\ell$ collinear points.

A similar result has been obtained independently by Cibulka and Kynčl [2]. Theorem 2 immediately implies that every point set with $\mathrm{ES}(8 \ell)$ points contains an empty pentagon or $\ell$ collinear points, which is already a substantial improvement on the result of Abel et al. [1 mentioned above. The rest of this section is dedicated to proving it. 


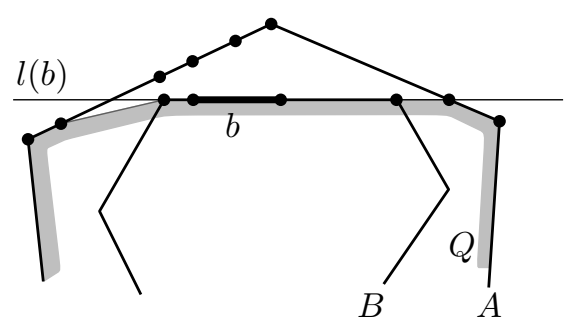

(a)

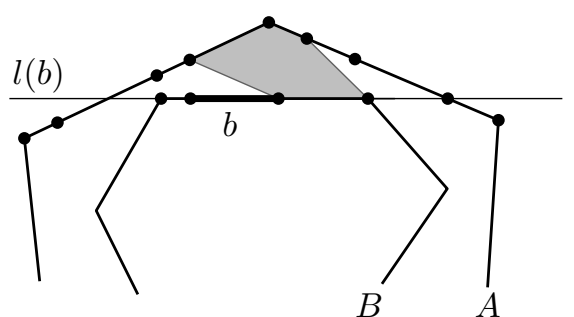

(b)

Figure 2. (a) If $\left|A \cap b^{+}\right| \leq|B \cap l(b)|$, then $A$ is not minimal. (b) If $b^{+}$ contained three non-collinear points of $A$, there would be an empty pentagon.

Throughout this section, let $P$ be a set of points in the plane that contains $8 \ell$ points in weakly convex position but contains no $\ell$ collinear points. Suppose for the sake of contradiction that $P$ contains no empty pentagon. Let $A$ be an inclusion-minimal weakly convex $8 \ell$-gon in $P$. That is, there is no weakly convex $8 \ell$-gon $A^{\prime}$ such that $\operatorname{conv}\left(A^{\prime}\right) \subsetneq \operatorname{conv}(A)$. An empty pentagon in $P \cap \operatorname{conv}(A)$ is an empty pentagon in $P$, so it can be assumed that $P \subseteq \operatorname{conv}(A)$, so $A$ is the first convex layer of $P$. Let $B$ be the second convex layer of $P$. For an edge $e$ of $A$ or $B$, let $e^{+}$be the open half-plane determined by $l(e)$ that does not contain any point in $B$.

Observation 3. For each edge $b$ of $B,\left|A \cap b^{+}\right|>|B \cap l(b)|$. Similarly, if $b_{1}, b_{2}, \ldots, b_{j}$ are edges of $B$, then

$$
\left|A \cap \bigcup_{i=1}^{j} b_{i}^{+}\right|>\left|B \cap \bigcup_{i=1}^{j} l\left(b_{i}\right)\right| .
$$

Proof. If $\left|A \cap b^{+}\right| \leq|B \cap l(b)|$ then removing the vertices $A \cap b^{+}$from $A$ and replacing them by $B \cap l(b)$ gives a weakly convex $m$-gon $Q$ such that $m \geq|A|$ and $\operatorname{conv}(Q) \subsetneq$ $\operatorname{conv}(A)$, contradicting the minimality of $A$; see Figure 2(a). The second claim follows from the minimality of $A$ in a similar way.

Observation 4. For each edge $b$ of $B$, the vertices of $A \cap b^{+}$are collinear.

Proof. By Observation 3 , there are at least 3 points in $A \cap b^{+}$. If $A \cap b^{+}$is not collinear, then there is an empty pentagon; see Figure 2(b).

The following lemma implies that $B$ has at least $4 \ell$ vertices.

Lemma 5. $2|B| \geq|A|$.

Proof. Since $|A| \geq 8 \ell, A$ has at least nine corners. Thus $B \neq \emptyset$. If $B$ is collinear then let $h$ be the line containing $B$. There are at most two corners of $A$ on $h$, so there are at least four corners of $A$ strictly to one side of $h$. The interior of the convex hull of these four corners together with any point in $B$ is empty. This implies that there is an empty pentagon in $P$, a contradiction.

Therefore $B$ has at least three corners, and at least three sides, where a side of $B$ is the set of edges between consecutive corners. Let $b_{1}, \ldots, b_{k}$ be edges of $B$, one in 


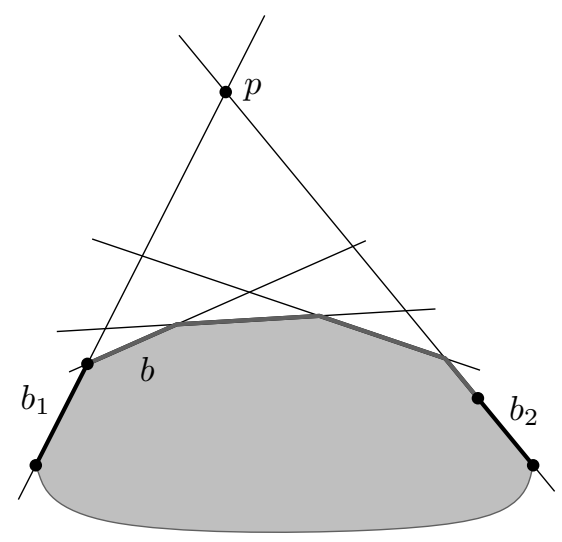

Figure 3. Lemma 6

each side of $B$. By Observation 4, each of the sets $A \cap b_{i}^{+}$is collinear for $i=1, \ldots, k$. Thus $|A| \leq \sum_{i=1}^{k}\left|A \cap b_{i}^{+}\right|<k \ell$, and so $k \geq 9$. In other words, $B$ has at least nine corners, so there is at least one point $z \in P$ in the interior of $\operatorname{conv}(B)$. Suppose that for some edge $x y$ of $A$ the closed triangle $\Delta[x, y, z]$ contains no point of $B$. Then there is an edge $x^{\prime} y^{\prime}$ of $B$ that crosses this triangle. The 4 -sector $S\left(x^{\prime}, x, y, y^{\prime}\right)$ contains $z$, contradicting the fact that $P$ contains no empty pentagon. Thus every such closed triangle contains a point of $B$. Since each point of $B$ is in at most two such closed triangles, $2|B| \geq|A|$.

The following lemma implies that for a set of points $X$, the first edge $b$ in $B$ in clockwise order such that $X \subseteq b^{+}$is well defined, as long as there is at least one such edge.

Lemma 6. For any set of points $X \neq \emptyset$, let $E_{X}$ be the set of edges $b$ in $B$ such that $X \subseteq b^{+}$. Then the edges in $E_{X}$ are consecutive in $B$, and not every edge of $B$ is in $E_{X}$.

Proof. If $X \cap \operatorname{conv}(B) \neq \emptyset$ then $E_{X}=\emptyset$. Take a point $x \in X$, so $x \notin \operatorname{conv}(B)$. Let $y$ be a point in the interior of $\operatorname{conv}(B)$ that is not collinear with any two points of $B \cup\{x\}$. Then $l(x y)$ intersects precisely two edges $b$ and $\tilde{b}$ of $B$, with $x \in b^{+}$and $x \in \tilde{b}^{-}$. Thus, $X \nsubseteq \tilde{b}^{+}$, so $E_{X}$ does not contain every edge of $B$.

If $E_{X}$ contains only one edge then the lemma holds, so consider two edges $b_{1}$ and $b_{2}$ in $E_{X}$ and suppose they are not consecutive. If $l\left(b_{1}\right)=l\left(b_{2}\right)$, then clearly the edges between $b_{1}$ and $b_{2}$ on $l\left(b_{1}\right)$ are also in $E_{X}$. Now suppose $l\left(b_{1}\right) \neq l\left(b_{2}\right)$. If $l\left(b_{1}\right)$ and $l\left(b_{2}\right)$ are parallel, then $b_{1}^{+} \cap b_{2}^{+}=\emptyset$, a contradiction. So $l\left(b_{1}\right)$ and $l\left(b_{2}\right)$ cross at a point $p$. Without loss of generality, $p$ is above $B$ with $b_{1}$ on the left and $b_{2}$ on the right, as shown in Figure 3. Let $b$ be the next edge clockwise from $b_{1}$. Then clearly $p \in b^{\oplus}$, so $b_{1}^{+} \cap b_{2}^{+} \subseteq b^{+}$, and hence $b \in E_{X}$. Iterating this argument shows that every edge clockwise from $b_{1}$ until $b_{2}$ is in $E_{X}$. It follows that the edges in $E_{X}$ are consecutive in $B$.

Let $a$ be an edge of $A$ such that $|A \cap l(a)| \geq 3$. Such an edge exists by Observations 3 and 4. Let $\left\{v_{1}, \ldots, v_{k}\right\}$ be $A \cap l(a)$ in clockwise order. Thus $k<\ell$. 


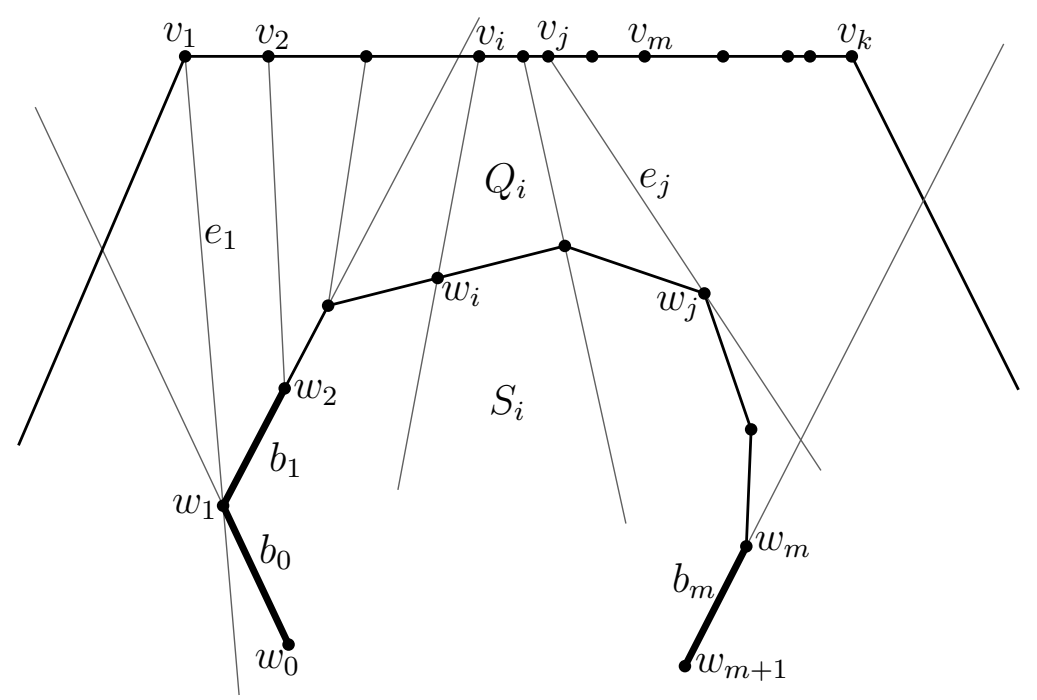

Figure 4. Definition of $b_{1}$ and the quadrilaterals $Q_{i}$.

Lemma 7. There is an edge $b$ of $B$ such that $\left\{v_{1}, v_{2}, v_{3}\right\} \subseteq b^{+}$or $\left\{v_{k-2}, v_{k-1}, v_{k}\right\} \subseteq$ $b^{+}$.

Proof. Let $b$ be an edge of $B$ with $v_{2} \in b^{+}$. Such an edge exists, since otherwise $v_{2} \in$ $\operatorname{conv}(B)$. Observations 3 and 4 imply that $\left|A \cap b^{+}\right| \geq 3$ and $A \cap b^{+}$is collinear. Thus if $v_{1} \in b^{+}$, then $\left\{v_{1}, v_{2}, v_{3}\right\} \subseteq b^{+}$, as required. Otherwise $l(b)$ intersects $l(a)$ between $v_{1}$ and $v_{2}$, so $\left\{v_{2}, v_{3}, \ldots, v_{k}\right\} \subseteq b^{+}$and $k \geq 4$, because if $k=3$ then $\left|A \cap b^{+}\right|=2$.

By Lemma 7. without loss of generality, there is an edge $b$ of $B$ such that $\left\{v_{1}, v_{2}, v_{3}\right\} \subseteq b^{+}$, and by Lemma 6 the edges with this property are consecutive in $B$. Let $b_{1}$ be the first one in clockwise order. For an illustration of the following definitions, see Figure 4. First observe that $\left|A \cap l(a) \cap \tilde{b}^{+}\right| \geq 3$ cannot hold for every edge $\tilde{b}$ of $B$, because otherwise $A \cap l(a)=A$ by Observation 4 , and so $|A|<\ell$. Define the endpoints of $b_{1}$ to be $w_{1}$ and $w_{2}$ in clockwise order. Let $w_{3}, \ldots, w_{m+1}$ and $b_{i}:=w_{i} w_{i+1}$ be subsequent vertices and edges of $B$ in clockwise order, where $\left|A \cap l(a) \cap b_{m-1}^{+}\right| \geq 3$ but $\left|A \cap l(a) \cap b_{m}^{+}\right| \leq 1$. Then $m \leq\left|B \cap \bigcup_{i=1}^{m-1} l\left(b_{i}\right)\right|<\left|A \cap \bigcup_{i=1}^{m-1} b_{i}^{+}\right| \leq k$ by Observation 3. Now define $e_{i}:=v_{i} w_{i}$ for $i=1, \ldots, m$. Let $e_{i}^{-}$be the open half-plane determined by $l\left(e_{i}\right)$ that contains $v_{1}$, or that does not contain $v_{2}$ in the case of $e_{1}$.

Let $j$ be minimal such that the closed half-plane $e_{j}^{\ominus}$ contains $B$. Clearly $j \neq 1$ since $w_{2} \in e_{1}^{+}$. The following argument shows that $j$ is well-defined. Call $e_{i}$ good if $w_{i}$ is the closest point of $l\left(e_{i}\right) \cap \operatorname{conv}(B)$ to $v_{i}$. First suppose that $e_{m}$ is good, so in particular $v_{m} \in b_{m-1}^{+}$. Since $m$ was chosen so that $\left|A \cap l(a) \cap b_{m-1}^{+}\right| \geq 3$ but $\left|A \cap l(a) \cap b_{m}^{+}\right| \leq 1$, and since $m<k$, it follows that $v_{m} \in b_{m}^{\ominus}$ also. This implies that $B \subseteq e_{m}^{\ominus}$, as illustrated in Figure $5(\mathrm{a})$, and so $j$ is well-defined. Now suppose that $e_{m}$ is not good. By the choice of $b_{1}$, both $e_{1}$ and $e_{2}$ are good, so let $p$ be minimal such that $e_{p}$ is not good. Thus $3 \leq p \leq m$. Then $w_{p-2}$ is in $e_{p-1}^{-}$because $e_{p-1}$ is good, and $w_{p}$ is in $e_{p-1}^{-}$because $e_{p}$ is not good, as shown in Figure 5(b). This implies that $B \subseteq e_{p-1}^{\ominus}$, so $j$ is well-defined. Note that this also shows that $e_{i}$ is good for all $i=1, \ldots, j$. 


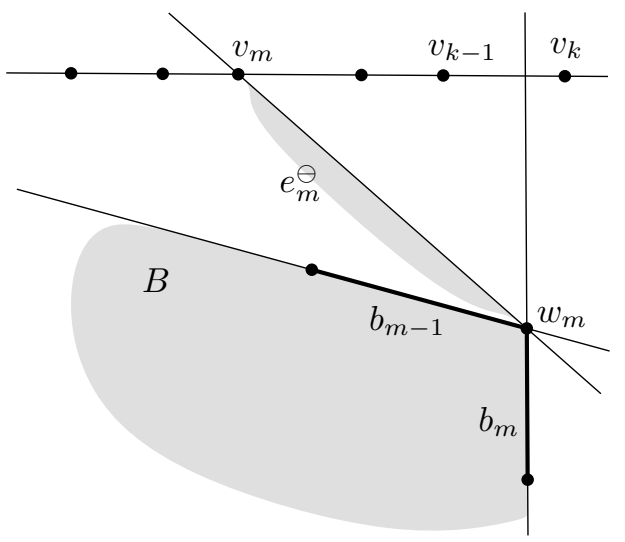

(a)

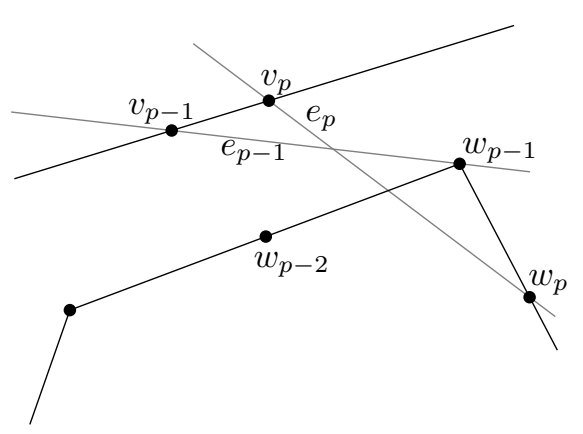

(b)

Figure 5. (a) If $e_{m}$ is good then $B \subseteq e_{m}^{\ominus}$. (b) If $e_{p-1}$ is good and $e_{p}$ is not, then $B \subseteq e_{p-1}^{\ominus}$.

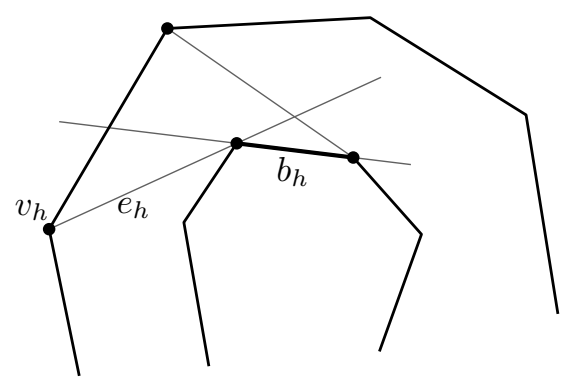

(a)

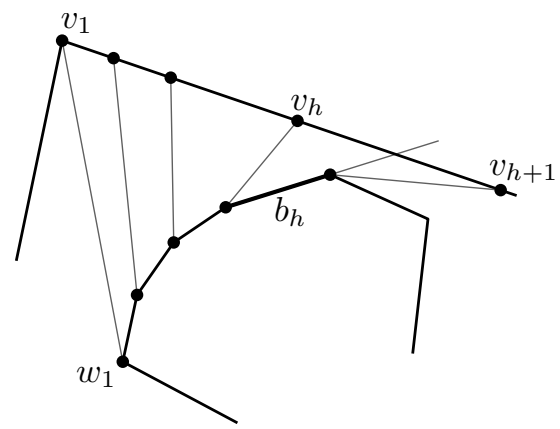

(b)

Figure 6. (a) If $v_{h} \in b_{h}^{\ominus}$ then $B \subseteq e_{h}^{\ominus}$. (b) If $v_{h+1} \in b_{h}^{\ominus}$ then $A \cap$ $\bigcup_{i=1}^{h} b_{i}^{+}=\left\{v_{1}, \ldots, v_{h}\right\}$.

Define the quadrilaterals $Q_{i}:=w_{i} v_{i} v_{i+1} w_{i+1}$ for $i=1, \ldots, j-1$. By the following argument, the quadrilaterals $Q_{i}$ are strictly convex. Suppose on the contrary that $Q_{h}$ is not strictly convex, and $h$ is minimal. There are two possible order types for $Q_{h}$. The first possibility is that $v_{h} \in b_{h}^{\ominus}$ and so $B \subseteq e_{h}^{\ominus}$ (since $e_{h}$ is good), contradicting the minimality of $j$; see Figure 6(a). The second possibility is that $v_{h+1} \in b_{h}^{\ominus}$ and so $A \cap \bigcup_{i=1}^{h} b_{i}^{+}=\left\{v_{1}, \ldots, v_{h}\right\}$, which contradicts Observation 3 since $\left|B \cap \bigcup_{i=1}^{h} l\left(b_{i}\right)\right| \geq h+1$; see Figure 6(b).

Let $S_{i}:=S\left[w_{i}, v_{i}, v_{i+1}, w_{i+1}\right]$ be the closed 4-sector of the quadrilateral $Q_{i}$ for $i=1, \ldots, j-1$. Note that $B \cap S_{i}=B \cap e_{i}^{\oplus} \cap e_{i+1}^{\ominus}$. Take a point $x \in B \cap e_{1}^{\oplus}$. Then $x \in e_{j}^{\ominus}$ since $B \subseteq e_{j}^{\ominus}$. Let $h$ be minimal such that $x \in e_{h+1}^{\ominus}$. If $h=0$ then $x \in l\left(e_{1}\right) \cap B \subseteq S_{1}$. Otherwise $x \notin e_{h}^{\ominus}$, so $x \in e_{h}^{\oplus}$, and so $x \in S_{h}$. Hence $B \cap e_{1}^{\oplus} \subseteq \bigcup_{i=1}^{j-1} S_{i}$.

The quadrilaterals $Q_{i}$ are empty because they lie between the layers $A$ and $B$. Therefore no $S_{i}$ contains a point of $B$ in its interior, and so all the points of $B \cap e_{1}^{\oplus}$ lie on the lines $l\left(e_{1}\right), \ldots, l\left(e_{j}\right)$. Since $B$ is in weakly convex position, $\left|B \cap l\left(e_{i}\right)\right| \leq 2$ for 


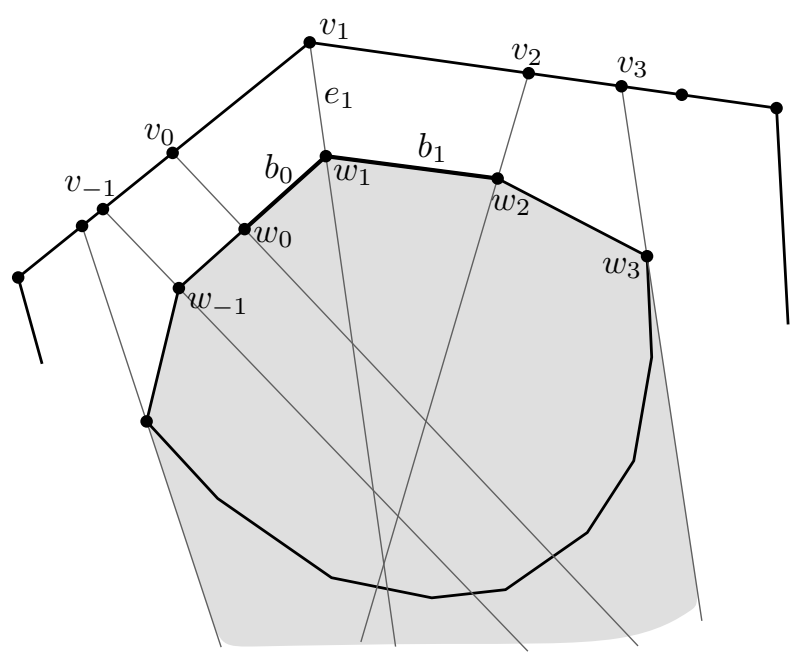

Figure 7. The convex hull of $B$ is covered by the union of the closed sectors $S_{i}$.

$i=2, \ldots, j-1$. There can be at most $\ell-2$ points of $B$ on $l\left(e_{1}\right)$ and $l\left(e_{j}\right)$. In fact there are less points of $B$ on $l\left(e_{1}\right)$ and $l\left(e_{j}\right)$, as the following argument shows. Note that $w_{m}$ is a corner of $B$ since $A \cap b_{m-1}^{+} \neq A \cap b_{m}^{+}$. Therefore $B \cap l\left(e_{j}\right) \subseteq\left\{w_{j}, \ldots, w_{m}\right\}$, and so $\left|B \cap l\left(e_{j}\right)\right| \leq m-j+1$. Since $j, m<\ell$, adding up the bounds for each $l\left(e_{i}\right)$ yields $\left|B \cap e_{1}^{\oplus}\right| \leq(\ell-2)+2(j-2)+(m-j+1)<3 \ell$. Since $|B| \geq 4 \ell$ by Lemma 5. this implies that $B \nsubseteq e_{1}^{\oplus}$, which implies that $\left|B \cap l\left(e_{1}\right)\right| \leq 2$. Hence $\left|B \cap e_{1}^{\oplus}\right| \leq 2(j-1)+(m-j+1)<2 \ell$.

It remains to bound the size of the rest of $B$, that is, $\left|B \cap e_{1}^{-}\right|$. Define $v_{0}, v_{-1}, v_{-2}, \ldots$ and $w_{0}, w_{-1}, w_{-2}, \ldots$ to be the vertices of $A$ and $B$ proceeding anticlockwise from $v_{1}$ and $w_{1}$ respectively. Define $b_{0}:=w_{0} w_{1}$. Since $B \nsubseteq e_{1}^{\oplus}$, it follows that $v_{1} \in b_{0}^{+}$, as shown in Figure 7. Since $b_{1}$ is the first edge in clockwise order with $\left\{v_{1}, v_{2}, v_{3}\right\} \subseteq$ $b_{1}^{+}$, neither $v_{2}$ nor $v_{3}$ is in $b_{0}^{+}$. Hence by Observation $3,\left\{v_{1}, v_{0}, v_{-1}\right\} \subseteq b_{0}^{+}$. Also, by Observation 4, neither $v_{0}$ nor $v_{-1}$ is in $b_{1}^{+}$, so $b_{0}$ is the first edge of $B$ with $\left\{v_{1}, v_{0}, v_{-1}\right\} \subseteq b_{0}^{+}$in anticlockwise order (recall that edges with this property are consecutive in $B$ by Lemma 7). Therefore, the argument that started at $b_{1}$ and proceeded clockwise may be started at $b_{0}$ and proceed anticlockwise instead. In this situation, the edge $e_{1}$ will remain the same as before because the starting points $v_{1}$ and $w_{1}$ are unchanged. Thus the argument will cover $B \cap e_{1}^{\ominus}$ with 4 -sectors and, analogously to before, show that $\left|B \cap e_{1}^{\ominus}\right|<2 \ell$. This implies that $|B| \leq\left|B \cap e_{1}^{\ominus}\right|+$ $\left|B \cap e_{1}^{\oplus}\right|<4 \ell$, which contradicts the fact that $|B| \geq 4 \ell$. This completes the proof of Theorem 2,

\section{Proof of Theorem 1}

Let $P$ be a set of at least $328 \ell^{2}$ points with no $\ell$ collinear points, and suppose for the sake of contradiction that $P$ does not contain an empty pentagon. Let $L_{1}, \ldots, L_{r}$ be the convex layers of $P$, with $L_{1}$ the outermost and $L_{r}$ the innermost layer. Theorem 2 implies that $\left|L_{i}\right|<8 \ell$ for every $i$. The layers are divided into three groups as follows. 
The layers $L_{r-\ell+1}$ to $L_{r}$ are the inner layers. Hence $\left|L_{r-\ell+1} \cup \cdots \cup L_{r}\right|<8 \ell^{2}$. The layers $L_{1}$ to $L_{a}$ are the outer layers, where $a$ is the minimum integer such that $\left|L_{1} \cup \cdots \cup L_{a}\right| \geq 64 \ell(\ell-1)$. This means that $\left|L_{1} \cup \cdots \cup L_{a}\right| \leq 64 \ell(\ell-1)+8 \ell<64 \ell^{2}$. The remaining layers $L_{a+1}$ to $L_{r-\ell}$ are the middle layers.

The strategy of the proof is to analyse the structure of the middle layers and show that if there are too many middle layers, then the outer layers contain less points than the lower bound in the previous paragraph. This contradiction implies that there are not too many middle layers. Since the size of each layer is limited by Theorem 2 , this yields an upper bound on the number of points in the middle layers. Adding this upper bound to those just established for the inner and outer layers will give a contradiction to the assumed size of $P$, completing the proof.

Abel et al. [1] introduced the following definition and lemma. Fix a point $z \in L_{r}$. An edge $x y$ of $L_{i}$ is empty if the open triangle $\Delta(x, y, z)$ contains no points of $L_{i+1}$.

Lemma 8. [1] If $L_{i}$ contains an empty edge for some $i \in\{1, \ldots, r-\ell+1\}$, then $P$ contains an empty pentagon or $\ell$ collinear points.

Lemma 8 is not stated in this form in the paper by Abel et al. [1], so the proof is included in Appendix $\mathrm{A}$ for completeness.

For now, consider only the points in the middle layers $L_{a+1}$ to $L_{r-\ell}$. For each point $v$ in a middle layer $L_{i}$, define the left and right child of $v$ as follows (see Figure 8(a)). Let $x$ be the closest point to $v$ in $\operatorname{conv}\left(L_{i+1}\right) \cap v z$ (where $v z$ is the line segment from $v$ to $z$ ). The right child of $v$ is the point in $L_{i+1}$ immediately clockwise from $x$. The left child of $v$ is the point in $L_{i+1}$ immediately anticlockwise from $x$. Note that although $x$ may be in $P, x$ is neither the left nor the right child of $v$.

A right chain is a sequence $v_{1}, \ldots, v_{t}$ of points in $L_{a+1} \cup \cdots \cup L_{r-\ell}$ such that $v_{i+1}$ is the right child of $v_{i}$. A left chain is defined in a similar fashion. A subchain is a chain contained in a larger chain, and a maximal chain is one that is not a proper subchain of another chain. A point cannot be the right child of two points $u$ and $v$ in $L_{i}$, otherwise the edge $u v$ (or the edges in the segment $u v$ if $u$ and $v$ are not adjacent) would be empty, contradicting Lemma 8 . Similarly, a point cannot be the left child of two points. This implies that maximal right chains do not intersect one another, and similarly for maximal left chains. Furthermore, by construction each point in the middle layers has a left and a right child, so every maximal chain contains a point in $L_{r-\ell}$. Together these observations imply the following lemma.

Lemma 9. Every point in the middle layers is in precisely one maximal right chain and one maximal left chain. The number of maximal right chains is $\left|L_{r-\ell}\right| \leq 8 \ell-1$, and similarly for maximal left chains.

The edges of a chain are the edges between consecutive vertices of the chain. A chain $V$ is said to wrap around if every ray starting at $z$ intersects the union of the edges of $V$ at least twice. Since chains advance in the same direction around $z$ with every step, this is equivalent to saying that $V$ covers a total angle of at least $4 \pi$ around $z$. 


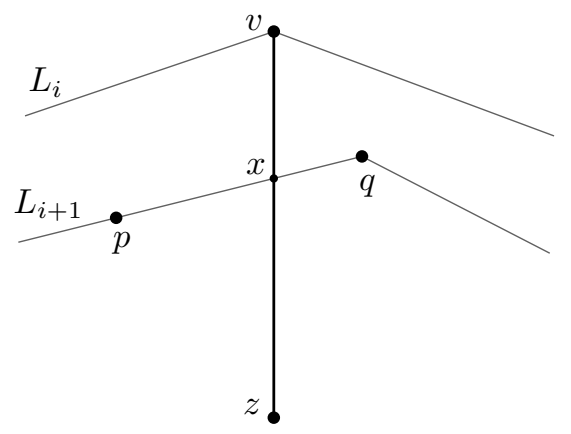

(a)

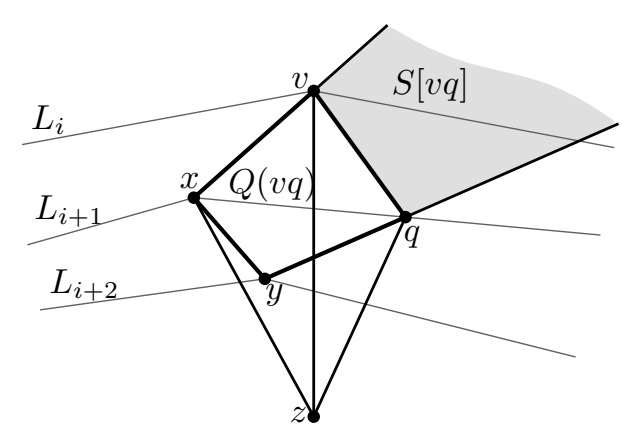

(b)

Figure 8. (a) The right child $q$ and the left child $p$ of $v$. (b) The quadrilateral $Q(v q)$ and the sector $S[v q]$.

Lemma 10. If the number of middle layers $r-\ell-a$ is at least $32 \ell$, then there is a chain with at most $32 \ell$ vertices that wraps around.

Proof. Let $V=\left(v_{1}, \ldots, v_{t}\right)$ be a right chain that starts at a point $v_{1} \in L_{a+1}$. Since $r-\ell-a \geq 32 \ell$, it can be assumed that $t=32 \ell$. By Lemma 9, each vertex $v_{i}$ lies in some left chain, and there are at most $8 \ell-1$ maximal left chains, so some left chain intersects $V$ at least five times. Let $U$ be a left chain that intersects $V$ in the points $p_{1}, \ldots, p_{5}$, where $p_{1}$ and $p_{5}$ are the first and last points of $U$ respectively.

Recall that right chains advance clockwise around $z$ with every step, and left chains anticlockwise. Therefore, the paths from $p_{i}$ to $p_{i+1}$ in $U$ and $V$ form a closed curve around $z$. So these paths cover an angle of $2 \pi$ around $z$. Hence $U$ and $V$ together cover a total angle of at least $8 \pi$ around $z$. This implies that at least one of them covers a total angle of at least $4 \pi$, and thus wraps around. Both $U$ and $V$ have at most $t$ vertices because they lie in the layers $L_{a+1}$ to $L_{a+t}$.

If $q$ is the right child of a vertex $v$ in a middle layer $L_{i}$, then associate with $v q$ the following quadrilateral, as illustrated in Figure 8(b). Let $x$ be the point in $L_{i+1}$ anticlockwise from $q$, so $x$ either lies on $v z$ or is the left child of $v$. Let $y$ be a point in the open triangle $\Delta(x, q, z)$ closest to $x q$. Such a $y$ exists in $L_{i+2}$, otherwise $x q$ would be an empty edge. Then $Q(v q):=v x y q$ is the quadrilateral associated with $v q$. This quadrilateral is strictly convex by construction. The triangle $\Delta[x, q, y]$ is empty since $x$ and $q$ are neighbours in $L_{i+1}$ and $y$ is a closest point to $x q$. The triangle $\Delta[v, q, x]$ is empty because it can contain neither a point of $L_{i}$ nor $L_{i+1}$. Thus $Q(v q)$ is an empty quadrilateral. Empty quadrilaterals determine 4-sectors that must be empty since there are no empty pentagons. Let $S[v q]$ be the closed 4 -sector determined by $Q(v q)$, that is, $S[v, x, y, q]$ in the notation established previously.

Let $V=\left(v_{1}, \ldots, v_{t}\right)$ be a chain and let $e_{i}:=v_{i} v_{i+1}$ be the edges of $V$. Let $e_{i}^{\oplus}$ be the closed half-plane defined by $e_{i}$ that does not contain $z$. Consider a quadrilateral $Q\left(e_{i}\right)=v_{i} x_{i} y_{i} v_{i+1}$ and let $c_{i}$ be the edge $x_{i} v_{i}$ and let $d_{i}$ be the opposite edge $y_{i} v_{i+1}$. Let $c_{i}^{\oplus}$ be $c_{i}^{\oplus}$ the closed half-plane defined by $c_{i}$ that contains $d_{i}$, and let $d_{i}^{\oplus}$ be the closed half-plane defined by $d_{i}$ that contains $c_{i}$. With these definitions, the 4 -sector defined by $Q\left(e_{i}\right)$ is $S\left[e_{i}\right]=c_{i}^{\oplus} \cap d_{i}^{\oplus} \cap e_{i}^{\oplus}$. 


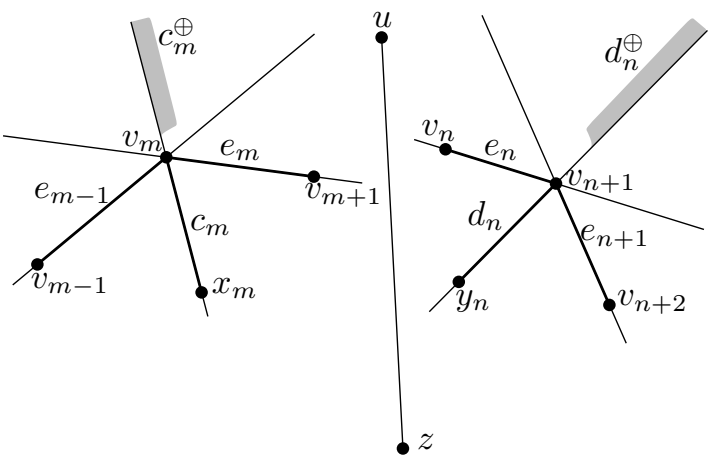

(a)

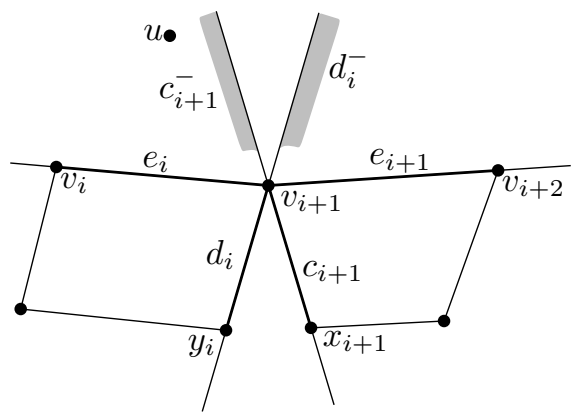

(b)

Figure 9. (a) $u \in c_{m}^{\oplus}$ and $u \in d_{n}^{\oplus}$. (b) $u$ cannot be in both $d_{i}^{-}$and $c_{i+1}^{-}$.

Lemma 11. If $V=\left(v_{1}, \ldots, v_{t}\right)$ wraps around, then the corresponding 4-sectors $S\left[e_{i}\right]$ cover the points of the outer layers $L_{1}$ to $L_{a}$.

Proof. Let $u$ be a point in $L_{1} \cup \cdots \cup L_{a}$. Without loss of generality, suppose that $V$ is a right chain, and that the line $l(u z)$ is vertical with $u$ above $z$. Consider the ray $h$ contained in $l(u z)$ that starts at $z$ and does not contain $u$. Since $V$ wraps around, it crosses $h$ at least twice. Therefore there are two non-consecutive edges $e_{j}$ and $e_{k}$ of $V$ that intersect $h$ (with $j<k$ ), and there is an edge $e_{p}$ between $e_{j}$ and $e_{k}$ that intersects the line segment $z u$.

Note that $u$ lies in $e_{j}^{-}$and $e_{k}^{-}$, but $u$ lies in $e_{p}^{+}$. Let $\tilde{V}$ be the maximal subchain of $V$ that contains $e_{p}$ and such that $u \in e^{+}$for every edge $e$ of $\tilde{V}$. Let $e_{m}$ and $e_{n}$ be the first and last edges of $\tilde{V}$. Since $e_{j}$ and $e_{k}$ are not in $\tilde{V}$ and $j<m \leq n<k$, the edges $e_{m-1}$ and $e_{n+1}$ are not in $\tilde{V}$. Thus $u \in e_{m-1}^{\ominus} \cap e_{m}^{+}$, as shown in Figure 9(a). Also, $v_{m}$ lies to the left of $l(u z)$ since $j<m \leq p$. This implies that $u$ and $v_{m+1}$ are on the same side of $l\left(c_{m}\right)$, so $u \in c_{m}^{\oplus}$. Furthermore, $u \in e_{n}^{+} \cap e_{n+1}^{\ominus}$, and $v_{n+1}$ lies to the right of $l(u z)$ since $p \leq n<k$, as shown in Figure 9(a) also. This implies that $u \in d_{n}^{\oplus}$.

Since $u \in e_{i}^{+} \cap e_{i+1}^{+}$for $m \leq i \leq n-1$, the fact that $y_{i}$ precedes $x_{i+1}$ in $L_{i+2}$ (or $y_{i}=x_{i+1}$ ) means that it is not possible for $u$ to be in both $d_{i}^{-}$and $c_{i+1}^{-}$; see Figure 9(b), In order to prove that $u$ is in some $S\left[e_{i}\right]=c_{i}^{\oplus} \cap d_{i}^{\oplus} \cap e_{i}^{\oplus}$, it suffices to show that $u \in c_{i}^{\oplus} \cap d_{i}^{\oplus}$ for some $i \in\{m, \ldots, n\}$. Let $q$ be minimal such that $u \in d_{q}^{\oplus}$. Such a $q$ exists because $u \in d_{n}^{\oplus}$. Then either $q=m$ or $u \in d_{q-1}^{-}$, so in any case $u \in c_{q}^{\oplus}$. Therefore $u$ lies in $S\left[e_{q}\right]$.

Lemma 10 says that if the number of middle layers $r-\ell-a$ is at least $32 \ell$, then there is a chain $V=\left(v_{1}, \ldots, v_{t}\right)$ with $t=32 \ell$ that wraps around. Since $P$ contains no empty pentagons, Lemma 11 then implies that every point in the outer layers lies on one of the lines $l\left(c_{i}\right)$ or $l\left(d_{i}\right)$ that bound the sectors $S\left[e_{i}\right]$ corresponding to $V$. Thus the number of points in the outer layers is at most $2 t(\ell-3)=64 \ell(\ell-3)$. Recall however that $a$ was chosen so that the outer layers contained at least $64 \ell(\ell-1)$ points, so in fact the number of middle layers is less than $32 \ell$. Therefore (by Theorem 2 the number of points in the middle layers is $\left|L_{a+1} \cup \cdots \cup L_{r-\ell}\right|<32 \ell \times 8 \ell=256 \ell^{2}$. As noted at the beginning of the proof, $\left|L_{1} \cup \cdots \cup L_{a}\right|<64 \ell^{2}$, and also $\left|L_{r-\ell+1} \cup \cdots \cup L_{r}\right|<8 \ell^{2}$. 
Adding everything up gives $|P|=\left|L_{1} \cup \cdots \cup L_{r}\right|<328 \ell^{2}$. This contradicts the assumption that $|P| \geq 328 \ell^{2}$, and so in fact $P$ does contain an empty pentagon. This completes the proof of Theorem 1 .

\section{Appendix A. Proof of Lemma 8}

Lemma 8 appears implicitly in the paper of Abel et al. [1]. The following proof is adapted directly from that paper, and the figures are reproduced with the kind permission of the authors. For simplicity, consider a point set $P$ with $\ell$ layers, so the statement becomes:

Lemma 8.1. Let $L_{1}, \ldots, L_{\ell}$ be the convex layers of a point set $P$. If $L_{1}$ contains an empty edge then $P$ contains an empty pentagon or $\ell$ collinear points.

Proof. Suppose for contradiction that $P$ contains no empty pentagon and no $\ell$ collinear points. Let $z$ be a point in the innermost layer $L_{\ell}$ of $P$. Suppose $x y$ is an empty edge of $L_{i}$ for some $i \in\{1, \ldots, \ell-2\}$. In this case, the intersection of the boundary of $\operatorname{conv}\left(L_{i+1}\right)$ and $\Delta(x, y, z)$ is contained in an edge $p q$ of $L_{i+1}$. Call $p q$ the follower of $x y$. First some properties of followers are established.

Claim 1. If $p q$ is the follower of $x y$, then $p x y q$ is an empty quadrilateral and $p q$ is empty.

Proof. Let $Q:=$ pxyq. Since $p$ and $q$ are in the interior of $\operatorname{conv}\left(L_{i}\right)$, both $x$ and $y$ are corners of $Q$. Both $p$ and $q$ are corners of $Q$, otherwise $x y$ would not be empty. Thus $Q$ is in strictly convex position. $Q$ is empty by the definition of $L_{i+1}$.

Suppose that $p q$ is not empty; that is, $\Delta(p, q, z) \cap L_{i+2} \neq \emptyset$. Then the 4-sector $S(p, x, y, q) \neq \emptyset$, so $P$ contains an empty pentagon. This contradiction proves that $p q$ is empty.

As illustrated in Figure 10(a)-(c), the follower $p q$ of $x y$ is said to be:

- double-aligned if $p \in l(x z)$ and $q \in l(y z)$,

- left-aligned if $p \in l(x z)$ and $q \notin l(y z)$,

- right-aligned if $p \notin l(x z)$ and $q \in l(y z)$.

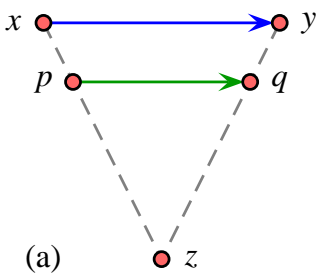

(a)

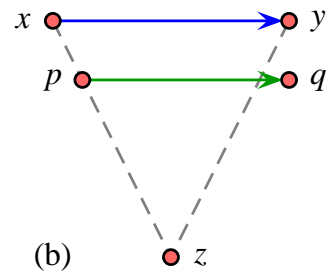

(b)

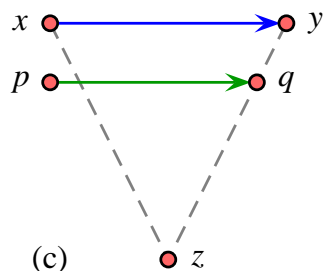

(c)

Figure 10. (a) Double-aligned. (b) Left-aligned. (c) Right-aligned.

Claim 2. If $p q$ is the follower of $x y$, then $p q$ is either double-aligned or left-aligned or right-aligned. 
Proof. Suppose that $p q$ is neither double-aligned nor left-aligned nor right-aligned, as illustrated in Figure 11(a). By Claim 1, pxyq is an empty quadrilateral. But the 4 -sector $S(p, x, y, q)$ contains the point $z$, so $P$ contains an empty pentagon.

Returning to the proof of Lemma 8, 1 , let $x_{1} y_{1}$ be the empty edge of $L_{1}$. For $i=$ $2,3, \ldots, \ell-1$, let $x_{i} y_{i}$ be the follower of $x_{i-1} y_{i-1}$. By Claim 1 (at each iteration), $x_{i} y_{i}$ is empty. For some $i \in\{2, \ldots, \ell-2\}$, the edge $x_{i} y_{i}$ is not double-aligned, as otherwise $\left\{x_{1}, x_{2}, \ldots, x_{\ell-2}, z\right\}$ are collinear and $\left\{y_{1}, y_{2}, \ldots, y_{\ell-2}, z\right\}$ are collinear, which implies that $\left\{x_{1}, x_{2}, \ldots, x_{\ell-1}, z\right\}$ are collinear or $\left\{y_{1}, y_{2}, \ldots, y_{\ell-1}, z\right\}$ are collinear by Claim 2 . Let $i$ be the minimum integer in $\{2, \ldots, \ell-2\}$ such that $x_{i} y_{i}$ is not double-aligned. Without loss of generality, $x_{i} y_{i}$ is left-aligned. On the other hand, $x_{j} y_{j}$ cannot be leftaligned for all $j \in\{i+1, \ldots, \ell-1\}$, as otherwise $\left\{x_{1}, x_{2}, \ldots, x_{\ell-1}, z\right\}$ are collinear. Let $j$ be the minimum integer in $\{i+1, \ldots, \ell-1\}$ such that $x_{j} y_{j}$ is not left-aligned. Thus $x_{j-1} y_{j-1}$ is left-aligned and $x_{j} y_{j}$ is not left-aligned. It follows that $x_{j-2} y_{j-2} y_{j-1} y_{j} x_{j-1}$ is an empty pentagon, as illustrated in Figure 11(b). This contradiction completes the proof.
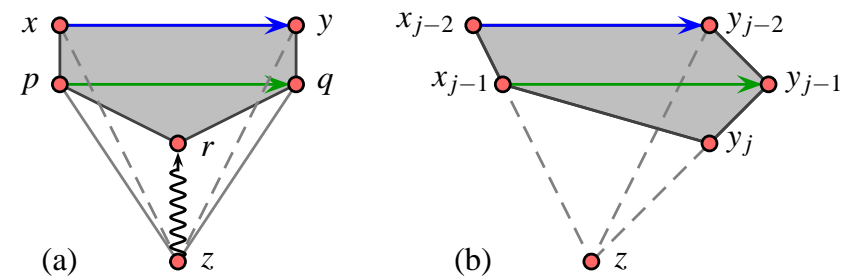

Figure 11. (a) Neither double-aligned nor left-aligned nor rightaligned. (b) The empty pentagon $x_{j-2} y_{j-2} y_{j-1} y_{j} x_{j-1}$.

\section{REFERENCES}

[1] Z. Abel, B. Ballinger, P. Bose, S. Collette, V. Dujmović, F. Hurtado, S. D. Kominers, S. Langerman, A. Pór, and D. R. Wood, Every large point set contains many collinear points or an empty pentagon, Graphs Combin., 27 (2011), pp. 47-60.

[2] J. Cibulka And J. KynčL, Private communication. 2012.

[3] D. Eppstein, Happy endings for flip graphs, J. Comput. Geom., 1 (2010), pp. 328.

[4] P. ERDős, On some problems of elementary and combinatorial geometry, Ann. Mat. Pura Appl. (4), 103 (1975), pp. 99-108.

[5] P. ERdös And G. Szekeres, A combinatorial problem in geometry, Compositio Math., 2 (1935), pp. 463-470.

[6] _ On some extremum problems in elementary geometry, Ann. Univ. Sci. Budapest. Eötvös Sect. Math., 3-4 (1960/1961), pp. 53-62.

[7] T. Gerken, Empty convex hexagons in planar point sets, Discrete Comput. Geom., 39 (2008), pp. 239-272. 
[8] H. Harborth, Konvexe Fünfecke in ebenen Punktmengen, Elem. Math., 33 (1978), pp. 116-118.

[9] J. D. Horton, Sets with no empty convex 7-gons, Canad. Math. Bull., 26 (1983), pp. $482-484$.

[10] V. A. Koshelev, The Erdős-Szekeres problem, Dokl. Akad. Nauk, 415 (2007), pp. $734-736$.

[11] C. M. Nicolás, The empty hexagon theorem, Discrete Comput. Geom., 38 (2007), pp. 389-397.

[12] S. Rabinowitz, Consequences of the pentagon property, Geombinatorics, 14 (2005), pp. 208-220.

[13] P. VAltr, On empty hexagons, in Surveys on discrete and computational geometry, vol. 453 of Contemp. Math., Amer. Math. Soc., 2008, pp. 433-441. 
School of Mathematical Sciences

MONASH UNIVERSITY

Victoria 3800, Australia

E-mail address: janos.barat@monash.edu

School of Computer Science

Carleton University

OtTaWa, Canada

E-mail address: vida@scs.carleton.ca

DÉPARTEMENT D'INFORMATIQUE

UNiversité LiBRE DE BRUXelles

Brussels, Belgium

E-mail address: gjoret@ulb.ac.be

Department of Mathematics and Statistics,

The University of Melbourne

Melbourne, Australia

E-mail address: m.payne3@pgrad.unimelb.edu.au

INSTITUT FÜR INFORMATIK

Freie UniversitätT BerLin

Berlin, Germany

E-mail address: scharf@mi.fu-berlin.de

INSTITUT FÜR INFORMATIK

Freie Universität BerLin

Berlin, Germany

E-mail address: daria.schymura@gmx.net

Department of Applied Mathematics and Institute for Theoretical Computer Science (CE-ITI),

Charles University

Prague, Czech Republic

Department of Mathematics and Statistics,

The University of Melbourne

Melbourne, Australia

E-mail address: woodd@unimelb.edu.au 